\title{
On the spectrum of the quadratic pencil of differential operators with periodic coefficients on the semi-axis
}

\section{Ashraf D Orujov*}

\section{"Correspondence:}

eorucov@cumhuriyet.edu.tr Department of Elementary Education, Cumhuriyet University,

Sivas, 58140, Turkey

\begin{abstract}
In this paper, the spectrum and resolvent of the operator $L_{\lambda}$ generated by the differential expression $\ell_{\lambda}(y)=y^{\prime \prime}+q_{1}(x) y^{\prime}+\left[\lambda^{2}+\lambda q_{2}(x)+q_{3}(x)\right] y$ and the boundary condition $y^{\prime}(0)-h y(0)=0$ are investigated in the space $L_{2}\left(\mathbb{R}^{+}\right)$. Here the coefficients $q_{1}(x), q_{2}(x), q_{3}(x)$ are periodic functions whose Fourier series are absolutely convergent and Fourier exponents are positive. It is shown that continuous spectrum of the operator $L_{\lambda}$ consists of the interval $(-\infty,+\infty)$. Moreover, at most a countable set of spectral singularities can exists over the continuous spectrum and at most a countable set of eigenvalues can be located outside of the interval $(-\infty,+\infty)$. Eigenvalues and spectral singularities with sufficiently large modulus are simple and lie near the points $\lambda= \pm \frac{n}{2}, n \in \mathbb{N}$.

MSC: 34L05; 47E05
\end{abstract}

Keywords: periodic; spectrum; resolvent; eigenvalue; spectral singularity

\section{Introduction}

In this study, the spectrum and resolvent of the maximal differential operator $L_{\lambda}$ generated by the linear differential expression

$$
\ell_{\lambda}(y)=y^{\prime \prime}+q_{1}(x) y^{\prime}+\left[\lambda^{2}+\lambda q_{2}(x)+q_{3}(x)\right] y
$$

and the boundary condition $y^{\prime}(0)-h y(0)=0$ have been investigated in the space $L_{2}\left(\mathbb{R}^{+}\right)$. Here $\lambda, h$ are complex parameters, $\mathbb{R}^{+}=(0,+\infty)$ and

$$
q_{j}(x)=\sum_{n=1}^{\infty} q_{j n} e^{i n x}, \quad j=1,2,3,
$$

with complex coefficients $q_{j n}$ for which

$$
\sum_{n=1}^{\infty} n\left|q_{j n}\right|<+\infty, \quad \sum_{n=1}^{\infty}\left|q_{3 n}\right|<+\infty, \quad j=1,2 .
$$

The domain of the operator $L_{\lambda}$ is

$$
\begin{aligned}
D\left(L_{\lambda}\right)= & \left\{y(x) \mid y(x), y^{\prime}(x) \in A C[0, R] \text { for all } R>0,\right. \\
& \left.y^{\prime}(0)-h y(0)=0, y(x), \ell_{\lambda}(y) \in L_{2}\left(\mathbb{R}^{+}\right)\right\} .
\end{aligned}
$$

(c) 2015 Orujov. This article is distributed under the terms of the Creative Commons Attribution 4.0 International License (http://creativecommons.org/licenses/by/4.0/), which permits unrestricted use, distribution, and reproduction in any medium, provided you give appropriate credit to the original author(s) and the source, provide a link to the Creative Commons license, and indicate if changes were made. 
Let $Q$ be the class of periodic functions $q(x)=\sum_{n=1}^{\infty} q_{n} e^{i n x}$ with $\|q(x)\|=\sum_{n=1}^{\infty}\left|q_{n}\right|<+\infty$, then $Q$ is a complex normed space and $q_{1}^{\prime}(x), q_{2}^{\prime}(x), q_{3}(x) \in Q$.

It is clear that if at least one of the functions $q_{j}(x), j=1,2,3$, is not zero, then the operator $L_{\lambda}$ is non-self-adjoint for each $\lambda \in \mathbb{C}$.

In the study [1], the Floquet solutions of equation $\ell_{\lambda}(y)=0$ in the case $q_{1}(x) \equiv q_{2}(x) \equiv 0$ were constructed, and using these solutions direct and inverse spectral problems were investigated for the operator $L=-\frac{d^{2}}{d x^{2}}+q(x)$ in the space $L_{2}(\mathbb{R})$. Later, using some different methods, the inverse problem for the operator $L=-\frac{d^{2}}{d x^{2}}+q(x)$ with periodic potential $q(x) \in L_{2}(0,2 \pi)$ was investigated in [2], the spectrum and resolvent operator were studied in [3]. Some results of [1] were generalized for the $2 n$ order linear differential operators with almost periodic coefficients from class $A P^{+}$of functions of the form $p(x)=\sum_{n=1}^{\infty} p_{n} e^{i \alpha_{n} x}$ with $\|p(x)\|=\sum_{n=1}^{\infty}\left|p_{n}\right|<+\infty$ in $[4,5]$. Here $\left(\alpha_{n}\right)_{n=1}^{\infty}$ is an increasing sequence of positive numbers and the set $\left\{\alpha_{n}: n \in \mathbb{N}\right\}$ is an additive semigroup. The spectrum and resolvent of a pencil of high order differential operators with periodic and almost periodic coefficients were investigated in [6] and [7, 8], respectively. The inverse problem for a pencil of $2 n$ order differential operators with periodic coefficients from the class $Q$ was studied in [9]. The pencil of the second order differential operators with periodic coefficients was investigated in $[10,11]$. Afterwards, the spectrum and resolvent for the pencil of the second order differential operators with almost periodic coefficients from the class $A P^{+}$under more general conditions on the coefficients were investigated in [12]. In all of these studies, the examined operators have a pure continuous spectrum, and the continuous spectrum consists of a half-line or union of lines pass from origin; moreover, there may be at most a countable number of spectral singularities on the continuous spectrum of the examined operators.

In the present study the operator $L_{\lambda}$ is investigated in the space $L_{2}\left(\mathbb{R}^{+}\right)$. It is proved that the continuous spectrum of the operator pencil $L_{\lambda}$ consists of the interval $(-\infty,+\infty)$ and there may be at most a countable set of spectral singularities on the continuous spectrum. Moreover, there may be a countable set $\sigma_{p}\left(L_{\lambda}\right)$ of eigenvalues outside the interval $(-\infty,+\infty)$, singular values $\lambda_{n}^{ \pm}$(eigenvalues or spectral singularities) with sufficiently large modulus are simple, lie in the neighborhood of points $\lambda= \pm \frac{n}{2}, n \in \mathbb{N}$ and satisfy the asymptotic formula

$$
\lambda_{n}^{ \pm}= \pm \frac{n}{2}+O\left(\frac{1}{n}\right), \quad n \rightarrow \infty
$$

Note that the analogous problem was investigated in [13] for the operator pencil $L_{\lambda}$ generated by the differential expression $\ell_{\lambda}(y)$ and the condition $y(0)=0$ in the space $L_{2}\left(\mathbb{R}^{+}\right)$ in case $q_{2}(x)=i q_{1}(x)$.

\section{Floquet solutions of the equation $\ell_{\lambda}(y)=0$}

The system of linear independent solutions of an equation of type $\ell_{\lambda}(y)=0$ with almost periodic coefficient from the class $A P^{+}$was investigated in [12]. According to Theorem 1 in the study [12], we can formulate the following theorem related with the equation

$$
y^{\prime \prime}+q_{1}(x) y^{\prime}+\left[\lambda^{2}+\lambda q_{2}(x)+q_{3}(x)\right] y=0, \quad-\infty<x<+\infty .
$$


Theorem 1 If the functions $q_{j}(x), j=1,2,3$, satisfy conditions (1) and (2), then for $\forall \lambda \in \mathbb{C}$, $\lambda \neq \pm \frac{n}{2}, n \in \mathbb{N}$ the differential equation (3) has the solutions

$$
f_{1}(x, \lambda)=e^{i \lambda x}\left(1+\sum_{n=1}^{\infty} U_{n}^{(1)}(\lambda) e^{i n x}\right), \quad f_{2}(x, \lambda)=e^{-i \lambda x}\left(1+\sum_{n=1}^{\infty} U_{n}^{(2)}(\lambda) e^{i n x}\right),
$$

where the series

$$
\sum_{n=1}^{\infty}\left|U_{n}^{(s)}(\lambda)\right| n^{2}, \quad s=1,2,
$$

is uniform convergent in each compact set $S \subseteq \mathbb{C}$ which does not contain the numbers $\lambda=$ $-\frac{n}{2}, n \in N$ in case $s=1$ and $\lambda=\frac{n}{2}, n \in \mathbb{N}$ in case $s=2$. Here $U_{n}^{(1)}(\lambda)=U_{n}^{(1)}+\sum_{k=1}^{n} \frac{U_{k n}^{(1)}}{k+2 \lambda}$, $U_{n}^{(2)}(\lambda)=U_{n}^{(2)}+\sum_{k=1}^{n} \frac{U_{k n}^{(2)}}{k-2 \lambda}, n \in \mathbb{N}$.

The solutions $f_{1}(x, \lambda)$ and $f_{2}(x, \lambda)$ can be used for the investigation of the structure of the spectrum and the kernel of the resolvent operator of $L_{\lambda}$, but they are not sufficient for studying the asymptotic of the singular values of the operator $L_{\lambda}$. For this reason it is convenient to use the Floquet solutions of the form

$$
\left\{\begin{array}{l}
f_{1}(x, \lambda)=e^{i \lambda x}\left(1+\sum_{n=1}^{\infty} U_{n}^{(1)} e^{i n x}+\sum_{k=1}^{\infty} \frac{1}{k+2 \lambda} \sum_{n=k}^{\infty} U_{k n}^{(1)} e^{i n x}\right) \\
f_{2}(x, \lambda)=e^{-i \lambda x}\left(1+\sum_{n=1}^{\infty} U_{n}^{(2)} e^{i n x}+\sum_{k=1}^{\infty} \frac{1}{k-2 \lambda} \sum_{n=k}^{\infty} U_{k n}^{(2)} e^{i n x}\right)
\end{array}\right.
$$

with conditions $\sum_{n=1}^{\infty} n^{2}\left|U_{n}^{(s)}\right|<+\infty, \sum_{k=1}^{\infty} \frac{1}{k} \sum_{n=k}^{\infty} n^{2}\left|U_{k n}^{(s)}\right|<+\infty, s=1,2$. It is clear that these representations of the solutions are a modified form of formulas (4).

The special solutions of type (5) are used in $[10,11]$ under various conditions on the coefficients of the considered equations. We use the following theorem about existence of the Floquet solutions of equation (3).

Theorem 2 If $q_{1}^{\prime}(x), q_{2}^{\prime}(x), q_{3}(x) \in Q$, then for each $\lambda \neq-\frac{n}{2}, n \in \mathbb{N}$ the differential equation (3) has solutions as

$$
f(x, \lambda)=e^{i \lambda x}\left(1+\sum_{n=1}^{\infty} u_{n} e^{i n x}+\sum_{k=1}^{\infty} \frac{1}{k+2 \lambda} \sum_{n=k}^{\infty} u_{k n} e^{i n x}\right),
$$

where the sequences $\left\{u_{n}\right\},\left\{u_{k n}\right\}$ of complex numbers are uniquely determined from the system of equations

$$
\left\{\begin{array}{l}
-n^{2} u_{n}-n \sum_{k=1}^{n} u_{k n}+q_{3 n}+\sum_{m=1}^{n-1}\left(i m q_{1, n-m}+q_{3, n-m}\right) u_{m} \\
\quad+\frac{1}{2} \sum_{m=1}^{n-1}\left(i q_{1, n-m}+q_{2, n-m}\right) \sum_{k=1}^{m} u_{k m}=0, \\
-2 n u_{n}+i q_{1 n}+q_{2 n}+\sum_{m=1}^{n-1}\left(i q_{1, n-m}+q_{2, n-m}\right) u_{m}=0, \quad n \in \mathbb{N}, \\
-n(n-k) u_{k n}+\sum_{m=k}^{n-1}\left[i m q_{1, n-m}+q_{3, n-m}-\frac{1}{2}\left(i q_{1, n-m}+q_{2, n-m}\right) k\right] u_{k m}=0, \\
\quad k, n \in \mathbb{N}, n \geq k+1
\end{array}\right.
$$

and the series

$$
\sum_{n=1}^{\infty} n^{2}\left|u_{n}\right|, \quad \sum_{k=1}^{\infty} \frac{1}{k} \sum_{n=k}^{\infty} n^{2}\left|u_{k n}\right|
$$

converge. 
Proof If we assume the existence of the solution of equation (3) of the form (6), according to convergence of series (8), we can find the derivatives of $f(x, \lambda)$ with respect to $x$ as follows:

$$
\begin{aligned}
& f^{\prime}(x, \lambda)=e^{i \lambda x}\left(i \lambda+\sum_{n=1}^{\infty} i(\lambda+n) u_{n} e^{i n x}+\sum_{k=1}^{\infty} \frac{1}{k+2 \lambda} \sum_{n=k}^{\infty} i(\lambda+n) u_{k n} e^{i n x}\right), \\
& f^{\prime \prime}(x, \lambda)=e^{i \lambda x}\left(-\lambda^{2}-\sum_{n=1}^{\infty}(\lambda+n)^{2} u_{n} e^{i n x}-\sum_{k=1}^{\infty} \frac{1}{k+2 \lambda} \sum_{n=k}^{\infty}(\lambda+n)^{2} u_{k n} e^{i n x}\right) .
\end{aligned}
$$

If we substitute these expressions in equation (3) and divide both sides by $e^{i \lambda x}$, according to uniqueness properties of the Fourier series, we have the following system of equations for the sequences $\left\{u_{n}\right\},\left\{u_{k n}\right\}$ :

$$
\begin{aligned}
& -\left(u_{n}+\sum_{k=1}^{n} \frac{u_{k n}}{k+2 \lambda}\right) n(n+2 \lambda)+i \lambda q_{1 n}+\lambda q_{2 n}+q_{3 n} \\
& \quad+\sum_{s+m=n}\left(u_{m}+\sum_{k=1}^{m} \frac{u_{k m}}{k+2 \lambda}\right)\left[i(\lambda+m) q_{1 s}+\lambda q_{2 s}+q_{3 s}\right]=0, \quad n \in \mathbb{N} .
\end{aligned}
$$

From this system we get the system of equations

$$
\left\{\begin{array}{l}
-n^{2} u_{n}-n \sum_{k=1}^{n} u_{k n}+q_{3 n}+\sum_{s+m=n}\left(i m q_{1 s}+q_{3 s}\right) u_{m} \\
\quad+\frac{1}{2} \sum_{s+m=n}\left(i q_{1 s}+q_{2 s}\right) \sum_{k=1}^{m} u_{k m}=0, \\
-2 n u_{n}+i q_{1 n}+q_{2 n}+\sum_{s+m=n}\left(i q_{1 s}+q_{2 s}\right) u_{m}=0, \quad n \in \mathbb{N}, \\
-n(n-k) u_{k n}+\sum_{\substack{s+m=n \\
m \geq k}}\left[i m q_{1 s}+q_{3 s}-\frac{1}{2}\left(i q_{1 s}+q_{2 s}\right) k\right] u_{k m}=0, \quad k, n \in \mathbb{N}, n \geq k+1
\end{array}\right.
$$

to determine $\left\{u_{n}\right\},\left\{u_{k n}\right\}$. The last system of equations can be rewritten as (7). Therefore the sequences $\left\{u_{n}\right\},\left\{u_{k n}\right\}$ in the expression of solution (6) of equation (3) satisfy system (7). On the contrary, if $\left\{u_{n}\right\},\left\{u_{k n}\right\}$ satisfy the system of equations (7) and series (8) converge, then the function $f(x, \lambda)$ determined by formula (6) is a solution of (3). Therefore to prove the theorem, it is sufficient to show the solvability of system (7) and the convergence of series (8). It is easy to see that the system of equations (7) has a unique solution. Indeed, from the second equation of system (7), the sequence $\left\{u_{n}\right\}$ is determined by the recurrent manner uniquely. Furthermore, by the known sequence $\left\{u_{n}\right\}$, from the first and third equations of system (7), the sequence $\left\{u_{k n}\right\}$ is also determined by the recurrent manner uniquely. It is not easy to prove the convergence of series (8) for the sequences determined from system (7). However, in the special case $q_{2}(x)=i q_{1}(x)$ this is proved in [13] (see Theorem 2). The existence of solution (6) can be proved by reducing to this special case. For this reason, we make the substitution $y(x)=z(x) e^{u(x)}$ in equation (3). Here, $z(x)$ is a new unknown function, $u(x)=-\frac{1}{2} \int\left[q_{1}(x)+i q_{2}(x)\right] d x=-\frac{1}{2} \sum_{n=1}^{\infty} \frac{2 q_{1 n}+i q_{2 n}}{i n} e^{i n x}$ is a twice continuously differentiable periodic function such that $u^{\prime}(x)=-\frac{1}{2}\left[q_{1}(x)+i q_{2}(x)\right]$ and $u(x), u^{\prime}(x), u^{\prime \prime}(x) \in Q$. If we take into account the expression of the function $y(x)$ in equation (3), we have

$$
z^{\prime \prime}+p(x) y^{\prime}+\left[\lambda^{2}+i \lambda p(x)+q(x)\right] y=0, \quad-\infty<x<+\infty,
$$


where $p(x)=-i q_{2}(x), q(x)=u^{\prime \prime}+\left(u^{\prime}\right)^{2}+q_{1}(x) u^{\prime}+q_{3}(x)=q_{3}(x)-\frac{1}{2}\left[q_{1}^{\prime}(x)+i q_{2}^{\prime}(x)\right]-\frac{1}{4}\left[q_{1}^{2}(x)+\right.$ $q_{2}^{2}(x)$ ]. Therefore, because of $p(x), p^{\prime}(x), q(x) \in Q$ by Theorem 2 in [13], we have that equation (9) has the solution of the form

$$
z(x, \lambda)=e^{i \lambda x}\left(1+\sum_{n=1}^{\infty} \widetilde{u}_{n} e^{i n x}+\sum_{k=1}^{\infty} \frac{1}{k+2 \lambda} \sum_{n=k}^{\infty} \widetilde{u}_{k n} e^{i n x}\right),
$$

where the series

$$
\sum_{n=1}^{\infty} n^{2}\left|\tilde{u}_{n}\right| \quad \text { and } \quad \sum_{k=1}^{\infty} \frac{1}{k} \sum_{n=k}^{\infty} n^{2}\left|\tilde{u}_{k n}\right|
$$

converge. Consequently,

$$
y(x, \lambda)=e^{u(x)} e^{i \lambda x}\left(1+\sum_{n=1}^{\infty} \widetilde{u}_{n} e^{i n x}+\sum_{k=1}^{\infty} \frac{1}{k+2 \lambda} \sum_{n=k}^{\infty} \widetilde{u}_{k n} e^{i n x}\right)
$$

is a solution of equation (3). Here, we can write $e^{u(x)}=1+u_{0}(x)$, where $u_{0}(x) \in Q$ by Wiener and Levy's theorem (see [14], p.34). Hence, we obtain

$$
\begin{aligned}
f(x, \lambda) & =y(x, \lambda)=e^{i \lambda x}\left(1+u_{0}(x)+\left(1+u_{0}(x)\right) \sum_{n=1}^{\infty} \widetilde{u}_{n} e^{i n x}+\sum_{k=1}^{\infty} \frac{1+u_{0}(x)}{k+2 \lambda} \sum_{n=k}^{\infty} \widetilde{u}_{k n} e^{i n x}\right) \\
& =e^{i \lambda x}\left(1+\sum_{n=1}^{\infty} u_{n} e^{i n x}+\sum_{k=1}^{\infty} \frac{1}{k+2 \lambda} \sum_{n=k}^{\infty} u_{k n} e^{i n x}\right),
\end{aligned}
$$

where $\sum_{n=1}^{\infty} u_{n} e^{i n x}=u_{0}(x)+\left(1+u_{0}(x)\right) \sum_{n=1}^{\infty} \tilde{u}_{n} e^{i n x} \in Q$ and $u_{k}(x)=\sum_{n=k}^{\infty} u_{k n} e^{i n x}=(1+$ $\left.u_{0}(x)\right) \sum_{n=k}^{\infty} \widetilde{u}_{k n} e^{i n x}$ with $u_{k}(x), u_{k}^{\prime}(x), u_{k}^{\prime \prime}(x) \in Q$ for $k \geq 1$. If we use the inequality $\|p(x) q(x)\| \leq\|p(x)\|\|q(x)\|, \forall p(x), q(x) \in Q$ which is satisfied in the normed space $Q$, the convergence of the series $\left\|\left(\sum_{n=1}^{\infty} u_{n} e^{i n x}\right)^{\prime \prime}\right\|=\sum_{n=1}^{\infty}\left|u_{n}\right| n^{2}$ and $\sum_{k=1}^{\infty} \frac{1}{k}\left\|u_{k}^{\prime \prime}(x)\right\|=\sum_{k=1}^{\infty} \frac{1}{k} \times$ $\sum_{n=k}^{\infty} n^{2}\left|u_{k n}\right|$ can be easily proved. Therefore we have that series (8) converge for solution $f(x, \lambda)$ of equation (3). The theorem is proved.

In order to find the second solution of equation (3) which is linearly independent with the solution $f(x, \lambda)$, we put $\mu=-\lambda$ in equation (3). Then equation (3) is written as

$$
y^{\prime \prime}+q_{1}(x) y^{\prime}+\left[\mu^{2}-\mu q_{2}(x)+q_{3}(x)\right] y=0, \quad-\infty<x<+\infty .
$$

By Theorem 2, equation (10) has the solution

$$
g(x, \mu)=e^{i \mu x}\left(1+\sum_{n=1}^{\infty} v_{n} e^{i n x}+\sum_{k=1}^{\infty} \frac{1}{k+2 \mu} \sum_{n=k}^{\infty} v_{k n} e^{i n x}\right) .
$$

Then, for each $\lambda \neq \frac{n}{2}, n \in \mathbb{N}$, the function

$$
\tilde{f}(x, \lambda)=g(x,-\lambda)=e^{-i \lambda x}\left(1+\sum_{n=1}^{\infty} v_{n} e^{i n x}+\sum_{k=1}^{\infty} \frac{1}{k-2 \lambda} \sum_{n=k}^{\infty} v_{k n} e^{i n x}\right)
$$


is the second solution of (3) where the series $\sum_{n=1}^{\infty}\left|v_{n}\right| n^{2}$ and $\sum_{k=1}^{\infty} \frac{1}{k} \sum_{n=k}^{\infty} n^{2}\left|v_{k n}\right|$ converge.

In what follows we use representations (5) for the solutions $f(x, \lambda), \widetilde{f}(x, \lambda)$.

Corollary 1 If the functions $q_{1}^{\prime}(x), q_{2}^{\prime}(x), q_{3}(x) \in Q$, then for $\forall \lambda \neq \pm \frac{n}{2}, \lambda \in \mathbb{C}, n \in \mathbb{N}$, equation (3) has the Floquet solutions

$$
\begin{aligned}
& f_{1}(x, \lambda)=e^{i \lambda x}\left(1+\sum_{n=1}^{\infty} U_{n}^{(1)} e^{i n x}+\sum_{k=1}^{\infty} \frac{1}{k+2 \lambda} \sum_{n=k}^{\infty} U_{k n}^{(1)} e^{i n x}\right), \\
& f_{2}(x, \lambda)=e^{-i \lambda x}\left(1+\sum_{n=1}^{\infty} U_{n}^{(2)} e^{i n x}+\sum_{k=1}^{\infty} \frac{1}{k-2 \lambda} \sum_{n=k}^{\infty} U_{k n}^{(2)} e^{i n x}\right),
\end{aligned}
$$

in $\mathbb{R}$ for which the series of type (8) converge.

Corollary 2 For each $x \in \mathbb{R}$, the functions $f_{j}(x, \lambda), j=1,2$, and their derivatives $f_{j}^{\prime}(x, \lambda)$, $f_{j}^{\prime \prime}(x, \lambda)$ with respect to $x$ are meromorphic functions with respect to $\lambda$, and they may have only simple poles $\lambda=(-1)^{j} n / 2, n \in \mathbb{N}$, and they are also continuous functions of pair $(x, \lambda)$ for all $x \in \mathbb{R}, \lambda \in \mathbb{C}, \lambda \neq(-1)^{j} n / 2, n \in \mathbb{N}$.

Corollary 3 The solutions $f_{1}(x, \lambda), f_{2}(x, \lambda)$ for $\forall \lambda \neq 0, \pm \frac{n}{2}, \lambda \in \mathbb{C}, n \in \mathbb{N}$ are linearly independent in each interval and their Wronskian is $W(x, \lambda)=W\left[f_{1}, f_{2}\right](x, \lambda)=-2 i \lambda e^{-\int q_{1}(x) d x}$, where $\int q_{1}(x) d x=\sum_{n=1}^{\infty} \frac{q_{1 n}}{i n} e^{i n x}$ (see [12], p.200).

Note that if at most one of the functions $q_{1}(x), q_{2}(x), q_{3}(x)$ is distinct from zero, then the functions $f_{j}(x, \lambda)$ have at most one pole. Namely, if $U_{n n}^{(j)} \neq 0$, then $\lambda=(-1)^{j} n / 2, j=1,2$, is the simple pole of the function $f_{j}(x, \lambda)$, if $U_{n n}^{(j)}=0$, then the function $f_{j}(x, \lambda)$ is regular at the point $\lambda=(-1)^{j} n / 2, j=1,2$.

The linearly independent solutions of equation (3) for $\lambda=0$ or $\lambda=\mp \frac{n}{2}, n \in \mathbb{N}$ have been constructed in [12]. These solutions are the functions $\widetilde{f}_{1 n}(x)=e^{-\frac{n}{2} x}\left(\psi_{1 n}(x)+x \phi_{1 n}(x)\right)$ and $f_{2}\left(x,-\frac{n}{2}\right)$ when $\lambda=-\frac{n}{2}, f_{1}\left(x, \frac{n}{2}\right)$ and $\tilde{f}_{2 n}(x)=e^{i \frac{n}{2} x}\left(\psi_{2 n}(x)+x \phi_{2 n}(x)\right)$ when $\lambda=\frac{n}{2}$, and $\tilde{f}_{1}(x)=$ $\psi_{0}(x), \tilde{f}_{2}(x)=x \psi_{0}(x)+\phi_{0}(x)$ when $\lambda=0$. Here the functions $\psi_{1 n}(x), \phi_{1 n}(x), \psi_{2 n}(x), \phi_{2 n}(x)$, $\psi_{0}(x), \phi_{0}(x)$ belong to the class $Q$.

\section{The spectrum and resolvent of the operator $L_{\lambda}$}

Theorem 3 The operator $L_{\lambda}$ does not have real eigenvalues, i.e. $\sigma_{p}\left(L_{\lambda}\right) \cap \mathbb{R}=\varnothing$.

Proof Let us show that the equation $L_{\lambda} y=0$ has only a trivial solution which belongs to $L_{2}\left(\mathbb{R}^{+}\right)$for $\forall \lambda \in \mathbb{R}$. In case $\lambda \neq 0, \pm \frac{n}{2}, n \in \mathbb{N}$ this follows from the properties of solutions $f_{1}(x, \lambda)$ and $f_{2}(x, \lambda)$. Indeed, if $y(x, \lambda)=c_{1} f_{1}(x, \lambda)+c_{2} f_{2}(x, \lambda)$ is the solution of the equation $l_{\lambda}(y)=0$ belonging to $L_{2}\left(\mathbb{R}^{+}\right)$and satisfying the condition $y^{\prime}(0)-h y(0)=0$, then $y(x, \lambda)$ is an almost periodic function and necessarily $y(x, \lambda) \equiv 0$, thus $\lambda$ is not eigenvalue. If linearly independent solutions of (3) according to $\lambda= \pm \frac{n}{2}, n \in \mathbb{N}$ or $\lambda=0$ are taken instead of $f_{1}(x, \lambda)$ and $f_{2}(x, \lambda)$, then similar result is also valid. Hence $\sigma_{p}\left(L_{\lambda}\right) \cap \mathbb{R}=\varnothing$. The theorem is proved.

Theorem 4 The operator $L_{\lambda}$ has at most a countable set of eigenvalues in $\mathbb{C} \backslash \mathbb{R}$. 
Proof It is easy to see that $f_{1}(x, \lambda) \in L_{2}\left(\mathbb{R}^{+}\right), f_{2}(x, \lambda) \notin L_{2}\left(\mathbb{R}^{+}\right)$for $\operatorname{Im} \lambda>0$ and $f_{1}(x, \lambda) \notin$ $L_{2}\left(\mathbb{R}^{+}\right), f_{2}(x, \lambda) \in L_{2}\left(\mathbb{R}^{+}\right)$for $\operatorname{Im} \lambda<0$. These relations imply

$$
y(x, \lambda)=c_{1} f_{1}(x, \lambda)+c_{2} f_{2}(x, \lambda) \in L_{2}(0,+\infty), \quad y^{\prime}(0, \lambda)-h y(0, \lambda)=0, \quad \lambda \in \mathbb{C} \backslash \mathbb{R},
$$

if and only if the eigenvalue equations

$$
\begin{array}{lll}
\Delta_{1}(\lambda)=f_{1}^{\prime}(0, \lambda)-h f_{1}(0, \lambda)=0 & \text { when } \operatorname{Im} \lambda>0 \quad \text { or } \\
\Delta_{2}(\lambda)=f_{2}^{\prime}(0, \lambda)-h f_{2}(0, \lambda)=0 & \text { when } \operatorname{Im} \lambda<0
\end{array}
$$

are satisfied.

Since $\Delta_{1}(\lambda)$ and $\Delta_{2}(\lambda)$ are holomorphic functions in the upper and lower half planes respectively, these equations have at most a countable set of roots in $\mathbb{C} \backslash \mathbb{R}$, i.e. $\sigma_{p}\left(L_{\lambda}\right)$ is a countable set. The theorem is proved.

Theorem 5 The residual spectrum of the operator $L_{\lambda}$ is an empty set, i.e. $\sigma_{r}\left(L_{\lambda}\right)=\varnothing$.

Proof Since the operator $L_{\lambda}$ is one to one for every $\lambda \in \mathbb{C} \backslash \sigma_{p}\left(L_{\lambda}\right)$, then $\lambda \in \sigma_{r}\left(L_{\lambda}\right)$ if and only if when the range $R\left(L_{\lambda}\right)$ is not dense in $L_{2}\left(\mathbb{R}^{+}\right)$. It means the equation $L_{\lambda}^{*}(z)=0$ has a nontrivial solution $z(x)$, in other words $\bar{\lambda} \in \sigma\left(L_{\lambda}^{*}\right)$. In here, if we take into account that the operator $L_{\lambda}^{*}$ is generated by the linear differential expression

$$
\ell_{\lambda}^{*}(z)=z^{\prime \prime}-\overline{q_{1}(x)} z^{\prime}+\overline{\left[\lambda^{2}+\lambda q_{2}(x)-q_{1}^{\prime}(x)+q_{3}(x)\right]} z
$$

and the boundary condition $z^{\prime}(0)-\overline{\left[h+q_{1}(0)\right]} z(0)=0$ in the domain

$$
\begin{aligned}
D\left(L_{\lambda}^{*}\right)= & \left\{z(x) \mid z(x), z^{\prime}(x)-\overline{q_{1}(x) z} \in A C[0, R] \text { for all } R>0,\right. \\
& \left.z^{\prime}(0)-\overline{\left[h+q_{1}(0)\right]} z(0)=0, z(x), \ell_{\lambda}^{*}(z) \in L_{2}\left(\mathbb{R}^{+}\right)\right\},
\end{aligned}
$$

there exists a nontrivial solution $\bar{z}(x) \in L_{2}\left(\mathbb{R}^{+}\right)$satisfying the condition $z^{\prime}(0)-\overline{\left[h+q_{1}(0)\right]} \times$ $z(0)=0$ of the conjugate equation

$$
z^{\prime \prime}(x)-q_{1}(x) z^{\prime}(x)+\left[\lambda^{2}+\lambda q_{2}(x)-q_{1}^{\prime}(x)+q_{3}(x)\right] z(x)=0, \quad 0<x<+\infty .
$$

Since equation (11) is an equation of type (3), then according to Theorem 2 equation (11) for $\lambda \in \mathbb{R}$ does not have a nontrivial solution $\bar{z}(x) \in L_{2}\left(\mathbb{R}^{+}\right)$, i.e. $\lambda \notin \sigma_{p}\left(L_{\lambda}^{*}\right)$ or $\sigma_{r}\left(L_{\lambda}\right) \cap \mathbb{R}=\varnothing$.

In general, if $\lambda \in \mathbb{C}, \lambda \neq 0, \pm \frac{n}{2}, n \in \mathbb{N}$, then according to Corollary 1 , equation (11) has the solutions as

$$
\varphi_{1}(x, \lambda)=e^{i \lambda x}\left(1+\sum_{n=1}^{\infty} V_{n}^{(1)} e^{i n x}+\sum_{k=1}^{\infty} \frac{1}{k+2 \lambda} \sum_{n=k}^{\infty} V_{k n}^{(1)} e^{i n x}\right)
$$

and

$$
\varphi_{2}(x, \lambda)=e^{-i \lambda x}\left(1+\sum_{n=1}^{\infty} V_{n}^{(2)} e^{i n x}+\sum_{k=1}^{\infty} \frac{1}{k-2 \lambda} \sum_{n=k}^{\infty} V_{k n}^{(2)} e^{i n x}\right)
$$


in $\mathbb{R}$. Clearly the functions (see [15], pp.208-210)

$$
\begin{aligned}
& z_{1}(x, \lambda)=-\frac{2 i \lambda f_{1}(x, \lambda)}{W\left[f_{1}, f_{2}\right](x, \lambda)}=-\frac{2 i \lambda f_{1}(x, \lambda)}{W(x, \lambda)}, \\
& z_{2}(x, \lambda)=-\frac{2 i \lambda f_{2}(x, \lambda)}{W\left[f_{1}, f_{2}\right](x, \lambda)}=-\frac{2 i \lambda f_{2}(x, \lambda)}{W(x, \lambda)}
\end{aligned}
$$

are linearly independent solutions of equation (11) for $\lambda \neq 0, \pm \frac{n}{2}, n \in \mathbb{N}$. By the uniqueness theorems for the analytic and almost periodic functions, it is easy to see that

$$
\varphi_{1}(x, \lambda)=-\frac{2 i \lambda f_{1}(x, \lambda)}{W(x, \lambda)}, \quad \varphi_{2}(x, \lambda)=-\frac{2 i \lambda f_{2}(x, \lambda)}{W(x, \lambda)} .
$$

From the explicit form of functions $\varphi_{i}(x, \lambda), i=1,2$, we have $\varphi_{1}(x, \lambda) \in L_{2}\left(\mathbb{R}^{+}\right), \varphi_{2}(x, \lambda) \notin$ $L_{2}\left(\mathbb{R}^{+}\right)$for $\operatorname{Im} \lambda>0$ and $\varphi_{1}(x, \lambda) \notin L_{2}\left(\mathbb{R}^{+}\right), \varphi_{2}(x, \lambda) \in L_{2}\left(\mathbb{R}^{+}\right)$for $\operatorname{Im} \lambda<0$. Consequently, the solution $\bar{z}(x)=c_{1} \varphi_{1}(x, \lambda)+c_{2} \varphi_{2}(x, \lambda)$ of equation (11) with conditions $\bar{z}^{\prime}(0)-\left[h+q_{1}(0)\right] \bar{z}(0)=$ 0 and $\bar{z}(x) \in L_{2}\left(\mathbb{R}^{+}\right)$only exists for values of the parameter $\lambda$ satisfying the equation $\varphi_{1}^{\prime}(0, \lambda)-[h+p(0)] \varphi_{1}(0, \lambda)=0$ when $\operatorname{Im} \lambda>0$ or the equation $\varphi_{2}^{\prime}(0, \lambda)-\left[h+q_{1}(0)\right] \varphi_{2}(0, \lambda)=$ 0 when $\operatorname{Im} \lambda<0$. From (12) we get

$$
\begin{aligned}
\varphi_{i}^{\prime}(x, \lambda)-\left[h+q_{1}(x)\right] \varphi_{i}(x, \lambda) \\
\quad=\left(-\frac{2 i \lambda f_{i}(x, \lambda)}{W(x, \lambda)}\right)^{\prime}+\left[h+q_{1}(x)\right] \frac{2 i \lambda f_{i}(x, \lambda)}{W(x, \lambda)} \\
\quad=-\frac{2 i \lambda f_{i}^{\prime}(x, \lambda) W(x, \lambda)-2 i \lambda f_{i}(x, \lambda) W^{\prime}(x, \lambda)}{(W(x, \lambda))^{2}}+\left[h+q_{1}(x)\right] \frac{2 i \lambda f_{i}(x, \lambda)}{W(x, \lambda)} \\
\quad=-\frac{2 i \lambda\left[f_{i}^{\prime}(x, \lambda)-h f_{i}(x, \lambda)\right]}{W(x, \lambda)}
\end{aligned}
$$

and

$$
\begin{aligned}
\varphi_{i}^{\prime}(0, \lambda)-\left[h+q_{1}(0)\right] \varphi_{i}(0, \lambda) & =-\frac{2 i \lambda\left[f_{i}^{\prime}(0, \lambda)-h f_{i}(0, \lambda)\right]}{W(0, \lambda)} \\
& =-\frac{2 i \lambda \Delta_{i}(\lambda)}{W(0, \lambda)}=\Delta_{i}(\lambda) e^{\sum_{n=1}^{\infty} \frac{q_{1 n}}{i n}}, \quad i=1,2 .
\end{aligned}
$$

Thus $\varphi_{i}^{\prime}(0, \lambda)-\left[h+q_{1}(0)\right] \varphi_{i}(0, \lambda)=0$ is equivalent to $\Delta_{i}(\lambda)=0, i=1,2$. By this, we have that if $\bar{\lambda} \in \mathbb{C} \backslash \mathbb{R}$ is an eigenvalue of the operator $L_{\lambda}^{*}$, then $\lambda$ is an eigenvalue of the operator $L_{\lambda}$. However it contradicts the fact that $\lambda \in \mathbb{C} \backslash \sigma_{p}\left(L_{\lambda}\right)$. Consequently, all $\lambda \in \mathbb{C} \backslash \mathbb{R}$ do not belong to residual spectrum of the operator $L_{\lambda}$, i.e. $\sigma_{r}\left(L_{\lambda}\right) \cap \mathbb{C} \backslash \mathbb{R}=\varnothing$. Since $\sigma_{r}\left(\mathbb{L}_{\lambda}\right) \cap \mathbb{R}=\varnothing$, consequently we get $\sigma_{r}\left(L_{\lambda}\right)=\varnothing$. The theorem is proved.

According to Theorem 5, for each $\lambda \in \mathbb{C} \backslash \sigma_{p}\left(L_{\lambda}\right)$, the inverse operator $L_{\lambda}^{-1}$ is defined in a dense subset of the space $L_{2}\left(\mathbb{R}^{+}\right)$. Let us show that for each $\lambda \in \mathbb{C} \backslash\left[\sigma_{p}\left(L_{\lambda}\right) \cup \mathbb{R}\right]$ the operator $L_{\lambda}^{-1}$ is bounded on $L_{2}\left(\mathbb{R}^{+}\right)$. For this reason, let us investigate the solution $y(x, \lambda) \in L_{2}\left(\mathbb{R}^{+}\right)$ of

$$
y^{\prime \prime}+q_{1}(x) y^{\prime}+\left[\lambda^{2}+\lambda q_{2}(x)+q_{3}(x)\right] y=f(x)
$$


satisfying the condition $y^{\prime}(0)-h y(0)=0$, where $f(x) \in L_{2}\left(\mathbb{R}^{+}\right)$. If we apply the Lagrange method by using Floquet solutions of equation (3), we find the solution of (13) as

$$
y(x, \lambda)=\int_{0}^{+\infty} G(x, t, \lambda) f(t) d t
$$

where

$$
G(x, t, \lambda)=\frac{1}{\Delta_{1}(\lambda) W(t, \lambda)} \begin{cases}f_{1}(x, \lambda) f_{0}(t, \lambda), & 0 \leq t<x, \\ f_{0}(x, \lambda) f_{1}(t, \lambda), & t \geq x\end{cases}
$$

if $\operatorname{Im} \lambda>0, \Delta_{1}(\lambda) \neq 0$,

$$
G(x, t, \lambda)=\frac{1}{\Delta_{2}(\lambda) W(t, \lambda)} \begin{cases}f_{2}(x, \lambda) f_{0}(t, \lambda), & 0 \leq t<x, \\ f_{0}(x, \lambda) f_{2}(t, \lambda), & t \geq x,\end{cases}
$$

if $\operatorname{Im} \lambda<0, \Delta_{2}(\lambda) \neq 0$, where

$$
f_{0}(x, \lambda)=\Delta_{2}(0) f_{1}(x, \lambda)-\Delta_{1}(0) f_{2}(x, \lambda)
$$

is the solution of equation (3) in $(-\infty,+\infty)$ with initial conditions $f_{0}(0, \lambda)=W(0, \lambda)=$ $-2 i \lambda w_{0}, f_{0}^{\prime}(0, \lambda)=-2 i \lambda h w_{0}, w_{0}=e^{-\sum_{n=1}^{\infty} \frac{q_{1 n}}{i n}}$. It is easy to see that the function

$$
\widehat{\varphi}(x, \lambda)=\frac{f_{0}(x, \lambda)}{W(x, \lambda)}=\frac{\Delta_{2}(0) \varphi_{1}(x, \lambda)-\Delta_{1}(0) \varphi_{2}(x, \lambda)}{-2 i \lambda}
$$

is the solution of equation (11) with the conditions $\widehat{\varphi}(0, \lambda)=1, \widehat{\varphi}^{\prime}(0, \lambda)=h+q_{1}(0)$ and the function

$$
\widehat{f}(x, \lambda)=\frac{f_{0}(x, \lambda)}{-2 i \lambda}=\frac{\Delta_{2}(0) f_{1}(x, \lambda)-\Delta_{1}(0) f_{2}(x, \lambda)}{-2 i \lambda}
$$

is the solution of equation (3) with the conditions $\widehat{f}(0, \lambda)=w_{0}, \widehat{f}^{\prime}(0, \lambda)=h w_{0}$. Therefore, these solutions are holomorphic functions of $\lambda$ in $\mathbb{C}$. Using these expressions we can write

$$
G(x, t, \lambda)=\frac{1}{\Delta_{1}(\lambda)} \begin{cases}f_{1}(x, \lambda) \widehat{\varphi}(t, \lambda), & 0 \leq t<x, \\ \widehat{f}(x, \lambda) \varphi_{1}(t, \lambda), & t \geq x\end{cases}
$$

if $\operatorname{Im} \lambda>0, \Delta_{1}(\lambda) \neq 0$,

$$
G(x, t, \lambda)=\frac{1}{\Delta_{2}(\lambda)} \begin{cases}f_{2}(x, \lambda) \widehat{\varphi}(t, \lambda), & 0 \leq t<x, \\ \widehat{f}(x, \lambda) \varphi_{2}(t, \lambda), & t \geq x\end{cases}
$$

if $\operatorname{Im} \lambda<0, \Delta_{2}(\lambda) \neq 0$. By these formulas the function $G(x, t, \lambda)$ is analytic with respect to $\lambda$ at point $\lambda$ for which $\Delta_{1}(\lambda) \neq 0$ and $\Delta_{2}(\lambda) \neq 0$. From the explicit expression of functions $f_{i}(x, \lambda)$ and $\varphi_{i}(x, \lambda)$ it follows that for $\forall \lambda \in \mathbb{C} \backslash \sigma_{p}\left(L_{\lambda}\right), \operatorname{Im} \lambda \neq 0$

$$
|G(x, t, \lambda)| \leq C(\lambda) e^{-\tau|x-t|},
$$


where $C(\lambda)>0, \tau=|\operatorname{Im} \lambda|, \forall x, t \in(0,+\infty)$. From here we have that

$$
\int_{0}^{+\infty}|G(x, t, \lambda)|^{2} d x<+\infty \text { and } \int_{0}^{+\infty}|G(x, t, \lambda)|^{2} d t<+\infty
$$

By considering formulas (14), (15) it can be proved by the standard method (see [16], pp.302-304) that for each $f(x) \in L_{2}\left(\mathbb{R}^{+}\right)$the function

$$
y(x, \lambda)=\int_{0}^{+\infty} G(x, t, \lambda) f(t) d t
$$

belongs to $L_{2}\left(\mathbb{R}^{+}\right)$and satisfies the condition $y^{\prime}(0, \lambda)-h y(0, \lambda)=0$. Further the integral operator $L_{\lambda}^{-1}: L_{2}\left(\mathbb{R}^{+}\right) \rightarrow L_{2}\left(\mathbb{R}^{+}\right)$defined by

$$
\left(R_{\lambda} f\right)(x)=\left(L_{\lambda}^{-1} f\right)(x)=\int_{0}^{+\infty} G(x, t, \lambda) f(t) d t
$$

is bounded for $\forall \lambda \in \mathbb{C} \backslash\left[\sigma_{p}\left(L_{\lambda}\right) \cup \mathbb{R}\right]$ and $\left\|R_{\lambda}\right\| \leq \frac{2|C(\lambda)|}{\tau}$. It means that $\lambda \in \rho\left(L_{\lambda}\right)$. On the other hand, for $\operatorname{Im} \lambda=0$, the operator $L_{\lambda}^{-1}$ is a closed operator defined on a dense proper subset of $L_{2}\left(\mathbb{R}^{+}\right)$, and so $L_{\lambda}^{-1}$ is an unbounded operator, which means $\lambda \in \sigma_{c}\left(L_{\lambda}\right)$.

It is clear that root $\lambda$ of the equations $\Delta_{1}(\lambda)=0, \operatorname{Im} \lambda \geq 0$ or $\Delta_{2}(\lambda)=0, \operatorname{Im} \lambda \leq 0$ may be a pole of the kernel $G(x, t, \lambda)$. If $\operatorname{Im} \lambda \neq 0$, then these poles are eigenvalues of the operator $L_{\lambda}$. If $\operatorname{Im} \lambda=0$, since $L_{\lambda}$ does not have $\lambda \in \mathbb{R}$ as an eigenvalue, then the kernel $G(x, t, \lambda)$ of the resolvent operator has poles at these points which are called spectral singularities (in the sense of [16], p.306) of the operator $L_{\lambda}$.

Thus the following theorem is true.

Theorem $6 L_{\lambda}$ has a continuous spectrum $\sigma_{c}\left(L_{\lambda}\right)=\mathbb{R}$, a countable set of eigenvalues $\sigma_{p}\left(L_{\lambda}\right) \subseteq \mathbb{C} \backslash \mathbb{R}$ and the resolvent set $\rho\left(L_{\lambda}\right)=\mathbb{C} \backslash\left(\mathbb{R} \cup \sigma_{p}\left(L_{\lambda}\right)\right)$. The resolvent operator $L_{\lambda}^{-1}$ is an integral operator in $L_{2}\left(\mathbb{R}^{+}\right)$with the kernel $G(x, t, \lambda)$ of Carleman type for $\lambda \in \rho\left(L_{\lambda}\right)$.

\section{The asymptotic formulas for singular values of the operator $L_{\lambda}$}

In this section we specify the location of the singular values of the operator $L_{\lambda}$ on the $\lambda$ complex plane and show that the singular values with sufficiently large modulus are located close to the points $\lambda= \pm \frac{n}{2}, n \in \mathbb{N}$. Note that by the singular values of the operator $L_{\lambda}$ we mean the eigenvalues and spectral singularities of the operator $L_{\lambda}$. For this reason we show that the singular values are located on the set $E_{\alpha}=\{\lambda|\lambda \in \mathbb{C},| \operatorname{Im} \lambda \mid<\alpha, \operatorname{Re} \lambda<\alpha\}$ for some $\alpha>1$. Let us prove this fact for the case $\operatorname{Im} \lambda \geq 0, \Delta_{1}(\lambda)=f_{1}^{\prime}(0, \lambda)-h f_{1}(0, \lambda)=0$; when $\operatorname{Im} \lambda \leq 0, \Delta_{2}(\lambda)=f_{2}^{\prime}(0, \lambda)-h f_{2}(0, \lambda)=0$, it is proved in a similar way.

Let $U_{0}(x)=1+\sum_{n=1}^{\infty} U_{n}^{(1)} e^{i n x}$. According to the second equation of system (7), the function $U_{0}(x)$ is the nontrivial solution of the equation $2 i y^{\prime}+\left[i q_{1}(x)+q_{2}(x)\right] y=0$, and so $U_{0}(x)=e^{\frac{1}{2} \int\left[i q_{2}(x)-q_{1}(x)\right] d x}, U_{0}=U_{0}(0)=1+\sum_{n=1}^{\infty} U_{n}^{(1)} \neq 0$. First, we show that there exists $\alpha>\max \left\{1,|h|, \sum_{k=1}^{\infty} \sum_{n=k}^{\infty}\left|U_{k n}^{(1)}\right| /\left|U_{0}\right|\right\}$ such that the equation $\Delta_{1}(\lambda)$ does not have a root outside the set $E_{\alpha}$. For this purpose, we use the representation

$$
\Delta_{1}(\lambda)=f_{1}^{\prime}(0, \lambda)-h f_{1}(0, \lambda)=(i \lambda-h) f_{1}(0, \lambda)+\sum_{n=1}^{\infty} i n U_{n}^{(1)}+\sum_{k=1}^{\infty} \frac{1}{k+2 \lambda} \sum_{n=k}^{\infty} i n U_{k n}^{(1)}
$$


Because of $|k+2 \lambda| \geq 2 \alpha>2$ and $|\lambda| \geq \alpha>|h|$ for each $\lambda \in \mathbb{C} \backslash E_{\alpha}, k \in \mathbb{N}$, the following inequalities

$$
\begin{aligned}
\left|\Delta_{1}(\lambda)\right| & \geq\left|(i \lambda-h) f_{1}(0, \lambda)\right|-\left|\sum_{n=1}^{\infty} i n U_{n}^{(1)}\right|-\left|\sum_{k=1}^{\infty} \frac{1}{k+2 \lambda} \sum_{n=k}^{\infty} i n U_{k n}^{(1)}\right| \\
& \geq(|\lambda|-|h|)\left|f_{1}(0, \lambda)\right|-\sum_{n=1}^{\infty} n\left|U_{n}^{(1)}\right|-\sum_{k=1}^{\infty} \frac{1}{|k+2 \lambda|} \sum_{n=k}^{\infty} n\left|U_{k n}^{(1)}\right| \\
& \geq(|\lambda|-|h|)\left|f_{1}(0, \lambda)\right|-\sum_{n=1}^{\infty} n\left|U_{n}^{(1)}\right|-\frac{1}{2} \sum_{k=1}^{\infty} \sum_{n=k}^{\infty} n\left|U_{k n}^{(1)}\right|
\end{aligned}
$$

and

$$
\begin{aligned}
\left|f_{1}(0, \lambda)\right| & =\left|1+\sum_{n=1}^{\infty} U_{n}^{(1)}+\sum_{k=1}^{\infty} \frac{1}{k+2 \lambda} \sum_{n=k}^{\infty} U_{k n}^{(1)}\right| \geq\left|1+\sum_{n=1}^{\infty} U_{n}^{(1)}\right|-\left|\sum_{k=1}^{\infty} \frac{1}{k+2 \lambda} \sum_{n=k}^{\infty} U_{k n}^{(1)}\right| \\
& \geq\left|U_{0}\right|-\sum_{k=1}^{\infty} \frac{1}{|k+2 \lambda|} \sum_{n=k}^{\infty}\left|U_{k n}^{(1)}\right| \geq\left|U_{0}\right|-\frac{1}{2 \alpha} \sum_{k=1}^{\infty} \sum_{n=k}^{\infty}\left|U_{k n}^{(1)}\right| \\
& >\left|U_{0}\right|-\frac{\left|U_{0}\right|}{2}=\frac{\left|U_{0}\right|}{2}
\end{aligned}
$$

are satisfied. According to above inequalities, we get

$$
\begin{aligned}
\left|\Delta_{1}(\lambda)\right| & >(|\lambda|-|h|) \frac{\left|U_{0}\right|}{2}-\sum_{n=1}^{\infty} n\left|U_{n}^{(1)}\right|-\frac{1}{2} \sum_{k=1}^{\infty} \sum_{n=k}^{\infty} n\left|U_{k n}^{(1)}\right| \\
& \geq \alpha \frac{\left|U_{0}\right|}{2}-|h| \frac{\left|U_{0}\right|}{2}-\sum_{n=1}^{\infty} n\left|U_{n}^{(1)}\right|-\frac{1}{2} \sum_{k=1}^{\infty} \sum_{n=k}^{\infty} n\left|U_{k n}^{(1)}\right|>0
\end{aligned}
$$

for each

$$
\alpha>\max \left\{1, \frac{\sum_{k=1}^{\infty} \sum_{n=k}^{\infty} n\left|U_{k n}^{(1)}\right|+2 \sum_{n=1}^{\infty} n\left|U_{n}^{(1)}\right|+|h|\left|U_{0}\right|}{\left|U_{0}\right|}\right\} .
$$

For some $\alpha$ satisfying this condition, the equation $\Delta_{1}(\lambda)=0$ does not have any root outside the set $E_{\alpha}$. On the other hand, the function $\Delta_{1}(\lambda)$ is holomorphic at every interior point $\lambda \neq-\frac{k}{2}(k \in \mathbb{N})$ of the set $E_{\alpha}$ and on the boundary of this set. Then the equation $\Delta_{1}(\lambda)=0$ may have at most a countable set of roots in the set $E_{\alpha}$, and all these roots may have the unique limit point at infinity. Let us show that the roots of the equation $\Delta_{1}(\lambda)=0$ with sufficiently large modulus are located close to the points $\lambda=-\frac{n}{2}, n \in \mathbb{N}$. For this, taking into account the absolute convergence of the series $\sum_{k=1}^{\infty} \sum_{n=k}^{\infty} n\left|U_{k n}^{(1)}\right|$, we can choose the smallest number $k_{0} \in \mathbb{N}, k_{0}>2$ such that $\sum_{k=k_{0}}^{\infty} \sum_{n=k}^{\infty} n\left|U_{k n}^{(1)}\right|<\frac{\left|U_{0}\right|}{4}$. Further, because of $\lim _{\lambda \rightarrow \infty} \sum_{k=1}^{k_{0}-1} \frac{1}{k+2 \lambda} \sum_{n=k}^{\infty} U_{k n}^{(1)}=0$, we can take the number $k_{1} \in \mathbb{N}, k_{1}>\max \left\{k_{0}, 3|h|+\frac{1}{2}\right\}$ such that the inequality

$$
\left|\sum_{n=1}^{k_{0}-1} \frac{1}{n+2 \lambda} \sum_{k=n}^{\infty} U_{n k}^{(1)}\right|<\frac{\left|U_{0}\right|}{4}
$$


is satisfied for all $r_{0} \geq k_{1}, r_{0} \in \mathbb{N}$ and all $\lambda \in \mathbb{C},|\operatorname{Im} \lambda|<\alpha, \operatorname{Re} \lambda \leq-\frac{r_{0}}{2}+\frac{1}{4}$. Thus, for $\lambda \in E_{\alpha}$, $\operatorname{Re} \lambda \leq-\frac{r_{0}}{2}+\frac{1}{4},|2 \lambda+k| \geq \frac{1}{3}, k \geq r_{0}$, we have $|\lambda|>\frac{3}{2}|h|,|2 \lambda+k| \geq \frac{1}{3}, k \geq 1$ and because of

$$
\begin{aligned}
\left|f_{1}(0, \lambda)\right| & \geq\left|U_{0}\right|-\left|\sum_{k=1}^{\infty} \frac{1}{k+2 \lambda} \sum_{n=k}^{\infty} U_{k n}^{(1)}\right| \\
& \geq\left|U_{0}\right|-\left|\sum_{k=1}^{k_{0}-1} \frac{1}{k+2 \lambda} \sum_{n=k}^{\infty} U_{k n}^{(1)}\right|-\sum_{k=k_{0}}^{\infty} \frac{1}{|k+2 \lambda|} \sum_{n=k}^{\infty}\left|U_{k n}^{(1)}\right| \\
& \geq\left|U_{0}\right|-\frac{\left|U_{0}\right|}{4}-\sum_{k=k_{0}}^{\infty} k \sum_{n=k}^{\infty}\left|U_{k n}^{(1)}\right|=\frac{3\left|U_{0}\right|}{4}-\sum_{k=k_{0}}^{\infty} \sum_{n=k}^{\infty} n\left|U_{k n}^{(1)}\right| \\
& >\frac{3\left|U_{0}\right|}{4}-\frac{\left|U_{0}\right|}{4}=\frac{\left|U_{0}\right|}{2}>0,
\end{aligned}
$$

we have the inequality

$$
\begin{aligned}
\left|\Delta_{1}(\lambda)\right| & \geq|i \lambda-h|\left|f_{1}(0, \lambda)\right|-\left|\sum_{n=1}^{\infty} i n U_{n}^{(1)}+\sum_{k=1}^{\infty} \frac{1}{k+2 \lambda} \sum_{n=k}^{\infty} i n U_{k n}^{(1)}\right| \\
& \geq \frac{\left|U_{0}\right|}{2}|i \lambda-h|-\sum_{n=1}^{\infty} n\left|U_{0 n}^{(1)}\right|-\sum_{k=1}^{\infty} \frac{1}{|k+2 \lambda|} \sum_{n=k}^{\infty} n\left|U_{k n}^{(1)}\right| \\
& \geq \frac{\left|U_{0}\right|}{2}|| \lambda|-| h||-\sum_{n=1}^{\infty} n\left|U_{n}^{(1)}\right|-3 \sum_{k=1}^{\infty} \sum_{n=k}^{\infty} n\left|U_{k n}^{(1)}\right| \\
& >\frac{\left|U_{0}\right|}{2}\left|\frac{r_{0}}{2}-\frac{1}{4}\right|-\frac{\left|U_{0}\right|}{2}|h|-\sum_{n=1}^{\infty} n\left|U_{n}^{(1)}\right|-3 \sum_{k=1}^{\infty} \sum_{n=k}^{\infty} n\left|U_{k n}^{(1)}\right|>0
\end{aligned}
$$

for

$$
\frac{r_{0}}{2}>\frac{1}{4}+\frac{\left|U_{0}\right||h|+2 \sum_{n=1}^{\infty} n\left|U_{n}^{(1)}\right|+6 \sum_{k=1}^{\infty} \sum_{n=k}^{\infty} n\left|U_{k n}^{(1)}\right|}{\left|U_{0}\right|}
$$

or for

$$
r_{0}>\frac{1}{2}+\frac{3\left|U_{0}\right||h|+4 \sum_{n=1}^{\infty} n\left|U_{n}^{(1)}\right|+12 \sum_{k=1}^{\infty} \sum_{n=k}^{\infty} n\left|U_{k n}^{(1)}\right|}{\left|U_{0}\right|} .
$$

Consequently, for the smallest $r_{0}>\max \left\{k_{1}, \frac{1}{2}+\frac{3\left|U_{0}\right||h|+4 \sum_{n=1}^{\infty} n\left|U_{n}^{(1)}\right|+12 \sum_{k=1}^{\infty} \sum_{n=k}^{\infty} n\left|U_{k n}^{(1)}\right|}{\left|U_{0}\right|}\right\}, r_{0} \in \mathbb{N}$, the roots of the equation $\Delta_{1}(\lambda)=0$ satisfying the conditions $\lambda \in E_{\alpha}$, Re $\lambda \leq-\frac{r_{0}}{2}+\frac{1}{4}$ can be located only in the neighborhood of the points $\lambda=-\frac{k}{2}, k \geq r_{0}$ with radius $\delta=\frac{1}{6}$. On the other hand, the equation $\Delta_{1}(\lambda)=0$ may have a finite number of roots satisfying the conditions $\lambda \in E_{\alpha}, \operatorname{Re} \lambda>-\frac{r_{0}}{2}+\frac{1}{4}$. Show that if the point $\lambda=-\frac{m}{2}, m \geq r_{0}$ is a pole of the function $f_{1}(0, \lambda)$, that is, $U_{m m}^{(1)} \neq 0$, then there is a unique simple root of $\Delta_{1}(\lambda)=0$ in the neighborhood $\left|\lambda+\frac{m}{2}\right|<\frac{1}{6}$. Indeed, the equations $\Delta_{1}(\lambda)=0$ and $(m+2 \lambda) \Delta_{1}(\lambda)=0$ have the same roots in the closed disk $\left|\lambda+\frac{m}{2}\right| \leq \frac{1}{6}$. Further if we put

$$
g(\lambda)=(m+2 \lambda)(i \lambda-h) U_{0}
$$




$$
h(\lambda)=(m+2 \lambda)\left((i \lambda-h) \sum_{k=1}^{\infty} \frac{1}{k+2 \lambda} \sum_{n=k}^{\infty} U_{k n}^{(1)}+\sum_{n=1}^{\infty} i n U_{n}^{(1)}+\sum_{k=1}^{\infty} \frac{1}{k+2 \lambda} \sum_{n=k}^{\infty} i n U_{k n}^{(1)}\right)
$$

then we have $\left|\lambda+\frac{k}{2}\right| \geq \frac{1}{6}$ for each $k \geq 1$ on the circle $\left|\lambda+\frac{m}{2}\right|=\frac{1}{6}$, and from (16) it is obtained that

$$
\begin{aligned}
|g(\lambda)| & |h(\lambda)| \\
= & |m+2 \lambda||i \lambda-h|\left|U_{0}\right|-|m+2 \lambda| \\
& \times\left|(i \lambda-h) \sum_{k=1}^{\infty} \frac{1}{k+2 \lambda} \sum_{n=k}^{\infty} U_{k n}^{(1)}+\sum_{n=1}^{\infty} i n U_{n}^{(1)}+\sum_{k=1}^{\infty} \frac{1}{k+2 \lambda} \sum_{n=k}^{\infty} i n U_{k n}^{(1)}\right| \\
\geq & \frac{1}{3}\left[|i \lambda-h|\left(\left|U_{0}\right|-\left|\sum_{k=1}^{\infty} \frac{1}{k+2 \lambda} \sum_{n=k}^{\infty} U_{k n}^{(1)}\right|\right)\right. \\
& \left.-\left|\sum_{n=1}^{\infty} i n U_{n}^{(1)}+\sum_{k=1}^{\infty} \frac{1}{k+2 \lambda} \sum_{n=k}^{\infty} i n U_{k n}^{(1)}\right|\right] \\
\geq & \frac{1}{3}\left((|\lambda|-|h|) \frac{\left|U_{0}\right|}{2}-\sum_{n=1}^{\infty} n\left|U_{n}^{(1)}\right|-\sum_{k=1}^{\infty} \frac{1}{|k+2 \lambda|} \sum_{n=k}^{\infty} n\left|U_{k n}^{(1)}\right|\right) \\
\geq & \frac{1}{3}\left(\left(\frac{r_{0}}{2}-\frac{1}{4}-|h|\right) \frac{\left|U_{0}\right|}{2}-\sum_{n=1}^{\infty} n\left|U_{n}^{(1)}\right|-\sum_{k=1}^{\infty} 3 \sum_{n=k}^{\infty} n\left|U_{k n}^{(1)}\right|\right)>0,
\end{aligned}
$$

i.e. $|g(\lambda)|>|h(\lambda)|$. Therefore, by the Rouche theorem, the functions $(m+2 \lambda) \Delta_{1}(\lambda)=g(\lambda)+$ $h(\lambda)$ and $g(\lambda)$ have the same number of zeros in the disk $\left|\lambda+\frac{m}{2}\right| \leq \frac{1}{6}$. Since the function $g(\lambda)$ has the unique simple zero $\lambda=-\frac{m}{2}$ in this disk, we have that the function $(m+2 \lambda) \Delta_{1}(\lambda)$ also has the unique simple zero $\lambda_{m}^{-}$in this disk. It is obvious that, if $\lambda=-\frac{m}{2}$ is not a pole of $f_{1}(0, \lambda)$, i.e. $U_{m m}^{(1)}=0$, then the equation $\Delta_{1}(\lambda)=0$ does not have any root in the disk $\left|\lambda+\frac{m}{2}\right| \leq \frac{1}{6}$. To show this it is sufficient to set

$$
\begin{aligned}
& g(\lambda)=(i \lambda-h) U_{0}, \\
& h(\lambda)=\left((i \lambda-h) \sum_{k=1}^{\infty} \frac{1}{k+2 \lambda} \sum_{n=k}^{\infty} U_{k n}^{(1)}+\sum_{n=1}^{\infty} i n U_{n}^{(1)}+\sum_{k=1}^{\infty} \frac{1}{k+2 \lambda} \sum_{n=k}^{\infty} i n U_{k n}^{(1)}\right)
\end{aligned}
$$

and apply the Rouche theorem to the function $\Delta_{1}(\lambda)=g(\lambda)+h(\lambda)$. Consequently, the equation $\Delta_{1}(\lambda)=0$ may have a unique simple root in the $\delta=\frac{1}{6}$-neighborhood of the point $\lambda=-\frac{m}{2}$ for all $m \geq r_{0}$. This implies that $\lambda_{m}^{-}=-\frac{m}{2}+\delta_{m},\left|\delta_{m}\right|<\frac{1}{6}, m \geq r_{0}$. We show that $\delta_{m}=O(1 / m), m \geq r_{0}$. It is easy to see that $\left|i \lambda_{m}^{-}-h\right| \geq\left|\lambda_{m}^{-}\right|-|h| \geq \frac{m}{2}-\left|\delta_{m}\right|-|h| \geq$ $\frac{m}{2}-\frac{1}{6}-|h| \geq m\left(\frac{1}{2}-\frac{1}{6 m}-\frac{|h|}{m}\right) \geq m\left(\frac{1}{2}-\frac{1}{18}-\frac{|h|}{3|h|}\right)=m\left(\frac{4}{9}-\frac{1}{3}\right)=\frac{m}{9}, m \geq r_{0}$. Now if we write the equation $\Delta_{1}\left(\lambda_{m}^{-}\right)=0$ in the form

$$
\begin{aligned}
& \left(i \lambda_{m}^{-}-h\right) U_{0}+\left(i \lambda_{m}^{-}-h\right) \sum_{k=1}^{\infty} \frac{1}{k+2 \lambda_{m}^{-}} \sum_{n=k}^{\infty} U_{k n}^{(1)}+\sum_{n=1}^{\infty} i n U_{n}^{(1)} \\
& +\sum_{k=1}^{\infty} \frac{1}{k+2 \lambda_{m}^{-}} \sum_{n=k}^{\infty} i n U_{k n}^{(1)}=0
\end{aligned}
$$


or

$$
\begin{aligned}
& \left(i \lambda_{m}^{-}-h\right)\left[U_{0}+\sum_{\substack{k=1 \\
k \neq m}}^{\infty} \frac{1}{k+2 \lambda_{m}^{-}} \sum_{n=k}^{\infty} U_{k n}^{(1)}\right]+\left(i \lambda_{m}^{-}-h\right) \frac{1}{m+2 \lambda_{m}^{-}} \sum_{n=m}^{\infty} U_{m n}^{(1)} \\
& \quad+\sum_{n=1}^{\infty} i n U_{n}^{(1)}+\sum_{k=1}^{\infty} \frac{1}{k+2 \lambda_{m}^{-}} \sum_{n=k}^{\infty} i n U_{k n}^{(1)}=0
\end{aligned}
$$

we find that

$$
\begin{aligned}
\mid m & +2 \lambda_{m}^{-}|| U_{0}+\sum_{\substack{k=1 \\
k \neq m}}^{\infty} \frac{1}{k+2 \lambda_{m}^{-}} \sum_{n=k}^{\infty} U_{k n}^{(1)} \mid \\
& \leq \sum_{n=m}^{\infty}\left|U_{m n}^{(1)}\right|+\frac{\left|m+2 \lambda_{m}^{-}\right|}{\left|i \lambda_{m}^{-}-h\right|} \sum_{n=1}^{\infty} n\left|U_{n}^{(1)}\right|+\frac{1}{\left|i \lambda_{m}^{-}-h\right|} \sum_{k=1}^{\infty} \frac{\left|m+2 \lambda_{m}^{-}\right|}{\left|k+2 \lambda_{m}^{-}\right|} \sum_{n=k}^{\infty} n\left|U_{k n}^{(1)}\right| \\
& \leq \frac{1}{m} \sum_{n=m}^{\infty} n\left|U_{m n}^{(1)}\right|+\frac{1 / 3}{m / 9} \sum_{n=1}^{\infty} n\left|U_{n}^{(1)}\right|+\frac{1}{m / 9} \sum_{k=1}^{\infty} \sum_{n=k}^{\infty} n\left|U_{k n}^{(1)}\right| \\
& \leq \frac{1}{m}\left\{\sum_{n=1}^{\infty} n\left|U_{m n}^{(1)}\right|+3 \sum_{n=1}^{\infty} n\left|U_{n}^{(1)}\right|+9 \sum_{k=1}^{\infty} \sum_{n=k}^{\infty} n\left|U_{k n}^{(1)}\right|\right\}
\end{aligned}
$$

or

$$
\left|2 \delta_{m}\right|\left|U_{0}+\sum_{\substack{k=1 \\ k \neq m}}^{\infty} \frac{1}{k+2 \lambda_{m}^{-}} \sum_{n=k}^{\infty} U_{k n}^{(1)}\right| \leq \frac{1}{m}\left\{\sum_{n=1}^{\infty} n\left|U_{m n}^{(1)}\right|+3 \sum_{n=1}^{\infty} n\left|U_{n}^{(1)}\right|+9 \sum_{k=1}^{\infty} \sum_{n=k}^{\infty} n\left|U_{k n}^{(1)}\right|\right\} .
$$

If here we take into account the estimation

$$
\begin{aligned}
& \left|U_{0}+\sum_{\substack{k=1 \\
k \neq m}}^{\infty} \frac{1}{k+2 \lambda_{m}^{-}} \sum_{n=k}^{\infty} U_{k n}^{(1)}\right| \\
& \geq\left|U_{0}\right|-\left|\sum_{\substack{k=1 \\
k \neq m}}^{\infty} \frac{1}{\left|k+2 \lambda_{m}^{-}\right|} \sum_{n=k}^{\infty} U_{k n}^{(1)}\right| \\
& \geq\left|U_{0}\right|-\left|\sum_{k=1}^{k_{0}-1} \frac{1}{k+2 \lambda_{m}^{-}} \sum_{n=k}^{\infty} U_{k n}^{(1)}\right|-\sum_{\substack{k=k_{0} \\
k \neq m}}^{\infty} \frac{1}{\left|k+2 \lambda_{m}^{-}\right|} \sum_{n=k}^{\infty}\left|U_{k n}^{(1)}\right| \\
& \geq\left|U_{0}\right|-\frac{\left|U_{0}\right|}{4}-\sum_{\substack{k=k_{0} \\
k \neq m}}^{\infty} 3 \sum_{n=k}^{\infty}\left|U_{k n}^{(1)}\right| \geq \frac{3\left|U_{0}\right|}{4}-\sum_{k=k_{0}}^{\infty} \sum_{n=k}^{\infty} n\left|U_{k n}^{(1)}\right|>\frac{3\left|U_{0}\right|}{4}-\frac{\left|U_{0}\right|}{4}=\frac{\left|U_{0}\right|}{2},
\end{aligned}
$$

we obtain

$$
\left|U_{0}\right|\left|\delta_{m}\right| \leq \frac{1}{m}\left\{\sum_{n=1}^{\infty} n\left|U_{m n}^{(1)}\right|+3 \sum_{n=1}^{\infty} n\left|U_{n}^{(1)}\right|+9 \sum_{k=1}^{\infty} \sum_{n=k}^{\infty} n\left|U_{k n}^{(1)}\right|\right\}
$$


or

$$
\left|\delta_{m}\right| \leq \frac{1}{m\left|U_{0}\right|}\left\{\sum_{n=1}^{\infty} n\left|U_{m n}^{(1)}\right|+3 \sum_{n=1}^{\infty} n\left|U_{n}^{(1)}\right|+9 \sum_{k=1}^{\infty} \sum_{n=k}^{\infty} n\left|U_{k n}^{(1)}\right|\right\}, \quad \forall m \geq r_{0}
$$

Since the series in brackets converge, the last inequality implies $\delta_{m}=O\left(\frac{1}{m}\right), \forall m \geq r_{0}$. Consequently, we prove that $\lambda_{m}^{-}=-\frac{m}{2}+O\left(\frac{1}{m}\right), \forall m \geq r_{0}$.

By a similar way, we can show that the equation $\Delta_{2}(\lambda)=0$ has the unique simple root $\lambda_{m}^{+}$in the $\delta=\frac{1}{6}$-neighborhood of the point $\lambda=\frac{m}{2}$ for all $m \geq r_{1}$ and some $r_{1} \in \mathbb{N}$ for which $\lambda_{m}^{+}=\frac{m}{2}+O\left(\frac{1}{m}\right), \forall m \geq r_{1}$ holds. Outside these neighborhoods, the equation $\Delta_{2}(\lambda)=0$ may have only a finite set of roots. If we set $m_{0}=\max \left\{r_{0}, r_{1}\right\}$, we have

$$
\lambda_{n}^{-}=-\frac{n}{2}+O\left(\frac{1}{n}\right), \quad \lambda_{n}^{+}=\frac{n}{2}+O\left(\frac{1}{n}\right), \quad n \geq m_{0} .
$$

Here $\lambda_{n}^{ \pm}$are the simple eigenvalues of the operator $L_{\lambda}$ if $\operatorname{Im} \lambda_{n}^{-}>0$ or $\operatorname{Im} \lambda_{n}^{+}<0$ and $f_{1}\left(x, \lambda_{n}^{-}\right)$, $f_{2}\left(x, \lambda_{n}^{+}\right)$are eigenfunctions corresponding to eigenvalues $\lambda_{n}^{ \pm} \cdot \operatorname{If} \operatorname{Im} \lambda_{n}^{-}=0$ or $\operatorname{Im} \lambda_{n}^{+}=0$, then $\lambda_{n}^{ \pm}$are the simple spectral singularities of the operator $L_{\lambda}$.

Taking into account the results of the work [13], it can easily be shown that all analysis of this work is also satisfied for the operator generated by the differential expression $\ell_{\lambda}(y)=$ $y^{\prime \prime}+q_{1}(x) y^{\prime}+\left[\lambda^{2}+\lambda q_{2}(x)+q_{3}(x)\right] y$ and the condition $y(0)=0$ in $L_{2}\left(\mathbb{R}^{+}\right)$. So the following theorem is true.

Theorem 7 The operator $L_{\lambda}$ generated by the linear differential expression $\ell_{\lambda}(y)=y^{\prime \prime}+$ $q_{1}(x) y^{\prime}+\left[\lambda^{2}+\lambda q_{2}(x)+q_{3}(x)\right] y$ and the boundary condition $y^{\prime}(0)-h y(0)=0($ or $y(0)=0)$ in the space $L_{2}\left(\mathbb{R}^{+}\right)$has the continuous spectrum $\sigma_{c}\left(L_{\lambda}\right)=\mathbb{R}$, may have at most a countable set of eigenvalues $\sigma_{p}\left(L_{\lambda}\right) \subseteq \mathbb{C} \backslash \mathbb{R}$ and a countable set of spectral singularities on the continuous spectrum. The resolvent operator $L_{\lambda}^{-1}$ is an integral operator in $L_{2}\left(\mathbb{R}^{+}\right)$with the kernel $G(x, t, \lambda)$ of the Carleman type for all $\lambda \in \rho\left(L_{\lambda}\right)=\mathbb{C} \backslash\left(\mathbb{R} \cup \sigma_{p}\left(L_{\lambda}\right)\right)$. Moreover, singular values $\lambda_{n}^{ \pm}$(eigenvalues or spectral singularities) with sufficiently large modulus are simple, lie in the neighborhood of points $\lambda= \pm \frac{n}{2}, n \in \mathbb{N}$ and the asymptotic formula

$$
\lambda_{n}^{ \pm}= \pm \frac{n}{2}+O\left(\frac{1}{n}\right), \quad n \rightarrow \infty
$$

is satisfied.

\section{Competing interests}

The author declares that they have no competing interests.

Received: 9 April 2015 Accepted: 22 June 2015 Published online: 14 July 2015

\section{References}

1. Gasymov, MG: Spectral analysis of a class of second-order non-self-adjoint differential operators. Funct. Anal. Appl. 14(1), 11-15 (1980) (Translated from Funkc. Anal. Prilozh 14(1), 14-19 (1980))

2. Pastur, LA, Tkachenko, VA: An inverse problem for a class of one-dimensional Shrödinger operators with a complex periodic potential. Math. USSR, Izv. 37(3), 611-629 (1991) (Translated from Izv. Akad. Nauk SSSR, Ser. Mat. 54(6), 1252-1269 (1990))

3. Shin, K: On half-line spectra for a class of non-self-adjoint Hill operators. Math. Nachr. 261/262, 171-175 (2003)

4. Gasymov, MG, Orudzhev, AD: Spectral properties of a class of differential operators with almost-periodic coefficients and their perturbations. Dokl. Akad. Nauk SSSR 287(4), 777-781 (1986) (in Russian)

5. Orudzhev, AD: Spectral analysis of a class of higher-order nonselfadjoint differential operators. Dokl. Akad. Nauk Azerb. SSR 37(2), 8-11 (1981) (in Russian) 
6. Orudzhev, EG: To spectral analysis of ordinary differential operators polynomially depending on a spectral parameter with periodic coefficients. Proc. Inst. Math. Mech. Natl. Acad. Sci. Azerb. 8(15), 169-175 (1998) (in Russian)

7. Orudzhev, EG: Investigation of the spectrum of a class of differential pencils with almost periodic coefficients. Dokl. Akad. Nauk Azerb. SSR 55(1-2), 27-31 (1999) (in Russian)

8. Orujov, AD: On the spectrum of the bundle of high order differential operators with almost periodic coefficients. Proc. Inst. Math. Mech. Natl. Acad. Sci. Azerb. 32(40), 165-174 (2010)

9. Efendiev, RF: The inverse problem for a class of ordinary differential operators with periodic coefficients. Zh. Mat. Fiz. Anal. Geom. 11(1), 114-121 (2004) (in Russian)

10. Efendiev, RF: An inverse problem for a class of second-order differential operators. Dokl. Akad. Nauk Azerb. SSR 57(4-6), 15-20 (2001) (in Russian)

11. Orudzhev, EG: On spectral decomposition by main functions of one quadratic bunch on the whole axis. Zh. Mat. Fiz. Anal. Geom. 12(1), 107-113 (2005) (in Russian)

12. Orujov, AD: On the spectrum of the bundle of second order differential operators with almost periodic coefficients. Int. J. Pure Appl. Math. 26(2), 195-204 (2006)

13. Orujov, AD: On the spectrum of the pencil of second order differential operators with periodic coefficients on the semi-axis. Proc. Inst. Math. Mech. Natl. Acad. Sci. Azerb. 40, Special Issue, 359-374 (2014)

14. Edwards, RE: Fourier Series: A Modern Introduction, vol. 2. Springer, New York (1982)

15. Stepanov, V: Course of Differential Equations. Fizmatgiz, Moscow (1959) (in Russian)

16. Naimark, MA: Linear Differential Operators. Frederick Ungar Publ. Co., New York (1968)

\section{Submit your manuscript to a SpringerOpen ${ }^{\mathcal{O}}$ journal and benefit from:}

- Convenient online submission

- Rigorous peer review

- Immediate publication on acceptance

Open access: articles freely available online

- High visibility within the field

- Retaining the copyright to your article 